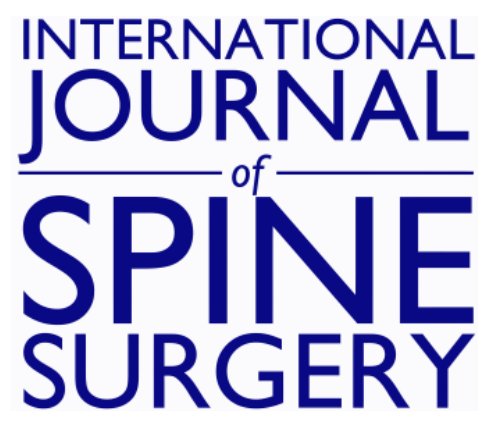

\title{
The Incidence of Venous Thromboembolism in Patients Undergoing Anterior Lumbar Interbody Fusion: A Proposed Thromboprophylactic Regime
}

Helen Vint, Matthew John Mawdsley, Christopher Coe, Cyrus D. Jensen and Ata G. Kasis

Int J Spine Surg 2021, 15 (2) 348-352

doi: https://doi.org/10.14444/8045

http://ijssurgery.com/content/15/2/348

This information is current as of April 26, 2023.

Email Alerts Receive free email-alerts when new articles cite this article. Sign up at:

http://ijssurgery.com/alerts

The International Journal of Spine Surgery

2397 Waterbury Circle, Suite 1,

Aurora, IL 60504, Phone: +1-630-375-1432 


\title{
The Incidence of Venous Thromboembolism in Patients Undergoing Anterior Lumbar Interbody Fusion: A Proposed Thromboprophylactic Regime
}

\author{
HELEN VINT, MBBS, FRCS(Tr\&Orth), ${ }^{1}$ MATTHEW JOHN MAWDSLEY, MBChB, PGCertClinEd, \\ FRCS(Tr\&Orth), ${ }^{2}$ CHRISTOPHER COE, MBChB, FRCA, FFFPM ${ }^{3}$ CYRUS D. JENSEN, MBBS, \\ FRCS(Tr\&Orth), ${ }^{2}$ ATA G. KASIS, MD, FRCS(Tr\&Orth) ${ }^{2}$ \\ ${ }^{I}$ Trauma and Orthopaedic Registrar, Northumbria Healthcare NHS Foundation Trust, Ashington, United Kingdom, ${ }^{2}$ Consultant Trauma and Orthopaedic Spinal \\ Surgeon, Northumbria Healthcare NHS Foundation Trust, Ashington, United Kingdom, ${ }^{3}$ Consultant Anaesthetist and Pain Specialist, Northumbria Healthcare \\ NHS Foundation Trust, Ashington, United Kingdom
}

\begin{abstract}
Objective: To determine the safety and efficacy of the proposed venous thromboembolism (VTE) prophylaxis regime in patients undergoing anterior lumbar interbody fusion (ALIF) surgery.

Background: Deep vein thrombosis (DVT) and pulmonary embolism (PE) are recognized complications after spine surgery, with rates in the literature ranging from $0 \%$ to $14 \%$ with some form of prophylaxis. Pharmacological thromboprophylaxis can cause postoperative bleeding and hematomas, which can result in significant neural compromise or permanent injury, and wound complications. ALIF surgery involves the handling and compression of major abdominal vessels during surgery and this adds to the risk of both arterial thrombosis and VTE.

Methods: A retrospective review of data, which were prospectively collected to evaluate the incidence of VTE in 200 consecutive patients undergoing ALIF following our VTE prophylaxis protocol. All patients had low molecular weight heparin, tinzaparin 4500 units subcutaneously on the evening before surgery, then daily for 3 to 5 days, then aspirin (acetylsalicylic acid) $150 \mathrm{mg}$ daily plus lansoprazole $30 \mathrm{mg}$ daily for 4 weeks after surgery. All patients had intermittent pneumatic compression of their calves and thighs intraoperatively and for 24 hours postoperatively then had early mobilization and thromboembolic deterrent stockings for 6 weeks.

Results: There was no incidence of any symptomatic VTE in the any of the 200 patients and no loss to follow-up. There was a $0 \%$ incidence of injury to the iliac vessels, symptomatic arterial occlusion, wound hematoma, major intraoperative bleeding, need for transfusion, symptomatic GI bleed, or retroperitoneal hematoma requiring intervention.

Conclusions: The proposed VTE prophylactic regime is safe and efficacious and may decrease the incidence of symptomatic VTE in patients undergoing an ALIF procedure, and despite the use of chemical thromboprophylaxis, there is no evidence of bleeding complications as a result of using this regime.
\end{abstract}

Level of Evidence: 4.

Complications

Keywords: ALIF, anterior lumbar interbody fusion, VTE, venous thromboembolism, prophylaxis, pulmonary embolism, deep vein thrombosis, aspirin, low molecular weight heparin, complication

\section{INTRODUCTION}

Deep vein thrombosis (DVT) and pulmonary embolism (PE) are recognized complications after spine surgery, with rates in the literature ranging from $0 \%$ to $14 \%$ with some form of prophylaxis. ${ }^{1-3}$ Venous thromboembolism (VTE) can result in associated morbidity and mortality and, as a consequence, numerous organizations have suggested guidelines to address the issue. ${ }^{4-6}$ However, there is a relative paucity of information available regarding the relative incidence of VTE complications for specific antithrombotic prophylactic measures within specific spine surgery patient subpopulations, such as patients undergoing anterior lumbar interbody fusion (ALIF) surgery. ${ }^{7}$ Furthermore, there is not enough data to definitively state the rate of clinically symptomatic DVT or PE for each type of spinal surgical intervention and the prophylactic measures that could be used, along with the risk to benefit ratio of those prophylactic measures.

There have been guidelines proposed to reduce the risk of VTE in orthopedic surgery, especially hip 
and knee replacement surgery and orthopedic trauma. ${ }^{4-6,8}$ Within the specialty of Spine Surgery, the British Association of Spinal Surgeons has proposed a method for stratifying patient risk in terms of their individual risk factors for bleeding and thrombosis risk along with the bleeding and thrombosis risk of the broad category of spinal procedure they are undergoing. From these data, they make general recommendations on the merits of use of mechanical and chemical prophylaxis, with a suggestion that all patients undergoing anterior lumbar surgery receive both mechanical prophylaxis and early addition of chemical prophylaxis unless bleeding risks are high. ${ }^{8}$ Due to the wide variation of both patient risk factors and individual spinal procedure, it is difficult to standardize a regime for all spinal surgery patients. Clearly the risks and rewards of prophylaxis must be considered for specific patients. Pharmacological thromboprophylaxis can cause postoperative bleeding and hematomas, which can result in significant neural compromise or permanent injury, and wound complications. ${ }^{9,10}$ It is for this reason that pharmacological thromboprophylactic treatment has never been met with universal acceptance from spine surgeons.

In terms of surgery specific risks, anterior lumbar spine surgery involves the handling and compression of major abdominal vessels during surgery and this adds to the risk of both arterial thrombosis and VTE. $^{11}$

To date, the guidelines available relating to VTE prophylaxis in elective anterior lumbar spinal surgery make only generalized recommendations with regard to mechanical and chemical prophylaxis, they are largely open to surgeon interpretation and preference, with no specific pharmacological regimes suggested. Herein we evaluate the incidence of VTE after ALIF using a combination of mechanical and pharmacological thromboprophylaxis preoperatively and postoperatively. We report the outcomes of adult patients undergoing ALIF and provide what the authors believe is the first ALIF-specific protocol for the perioperative prevention of VTE in the published literature. This regime was adopted from Gold Coast Spine, Australia.

\section{METHODS}

A retrospective review of data that were prospectively collected to evaluate the incidence of VTE in patients undergoing ALIF following our treatment protocol and to assess its efficacy in prevention and associated complications. We identified 200 consecutive patients who underwent ALIF for degenerative conditions from 2013 to 2017.

A standard left (or right) sided mini-open anterior retroperitoneal approach was used; an Omni-Tract (Omni-Tract Surgical) Wishbone Style table-mounted abdominal retractor was used to help retract the abdominal contents. Lateral retraction of the left and right common iliac vessels was performed for the L4/5 level and above with pins into the vertebral body. For the L5/S1 level, the vessels were moved laterally, and pins were inserted into the vertebral body. As a general principle, the operating surgeon attempted to minimize the time where the vessels were under retraction; however, there was no effort to temporarily release the pressure on the vessels during the procedure to intermittently restore blood flow as some surgeons advocate. Care was taken to handle the vessels very carefully to minimize the risk of injury and thrombotic events.

The standard procedure for all cases was to use perioperative cell salvage and re-transfuse the patients if there was significant bleeding. All patients had low molecular weight heparin (LMWH), tinzaparin 4500 units (Innohep; LEO Pharma A/S, Ballerup, Denmark), subcutaneously on the evening before surgery, then daily for 3 to 5 days (whilst an in-patient) and then aspirin (acetylsalicylic acid) $150 \mathrm{mg}$ daily plus lansoprazole 30 mg daily for 4 weeks after surgery. All patients wore thromboembolic deterrent stockings (TEDS) for a total of 6 weeks from the time of surgery. All patients had intermittent pneumatic compression of their calves and thighs (Flotron) intraoperatively and for 24 hours postoperatively. Patients were mobilized the morning after surgery.

All patients were routinely reviewed in the outpatient clinic at 2, 6, 24, and 52 weeks. The records of all patients were reviewed for the incidence of VTE. All patients were asked specifically at their 6-month review point if they had undergone any VTE investigations or treatments, to identify any patients who might have had a VTE in between clinic appointments or in other hospitals.

\section{RESULTS}

Two hundred consecutive patients were included in the study. The average age was $44.6(28-70.3)$ 
Table 1. Level of anterior lumbar interbody fusion procedure.

\begin{tabular}{lcc}
\hline Level & Number of Patients & Percentage \\
\hline L3/4 & 1 & 0.5 \\
L4/5 & 77 & 38.5 \\
L5/S1 & 106 & 53 \\
L3/4 and L4/5 & 1 & 0.5 \\
L4/5 \& L5/S1 & 14 & 7 \\
L3/4, L4/5, and L5/S1 & 1 & 0.5 \\
$\quad$ Total & 200 & \\
\hline
\end{tabular}

years. One hundred eighteen patients were female. The ALIF was performed for a single level in 184 patients, mostly at L4/5 or L5/S1 (see Table 1). One of the patients had ALIF at L5/S1 that was a revision procedure of a previous ALIF.

The most common indication for surgery was neuroforaminal stenosis caused by loss of disc height in 59 patients (see Table 2). Fourteen patients had previously had a posterior approach and spinal fusion and the ALIF was performed due to ongoing pain and nonunion. All 61 patients with spondylolisthesis or with a failed posterior fusion had a combined procedure of both an ALIF and a posterior stabilization at the same sitting.

There was no incidence of any symptomatic VTE in the any of the 200 patients, and there was no loss to follow-up. There was a $0 \%$ incidence of injury to the iliac vessels, symptomatic arterial occlusion, wound hematoma, major intraoperative bleeding, need for transfusion, and no symptomatic retroperitoneal hematoma requiring intervention. There were no symptomatic GI bleeds. There were 2 superficial wound infections treated with oral antibiotics, one of which required a negative pressure wound therapy dressing for 10 days.

\section{DISCUSSION}

This review of patients reveals a $0 \%$ incidence of clinically detectable VTE in our patient sample, an incidence that is lower than the $0.4 \%$ to $2.4 \%$ in the published literature following ALIF surgery. ${ }^{10,12-16}$ A large retrospective analysis of 1474 patients undergoing single-level ALIF surgery registered in the American College of Surgeons National Surgical Quality Improvement database suggests a $0.8 \%$ postoperative risk of $\mathrm{PE}$ and $0.9 \%$ risk of DVT requiring therapy. No further information is available on the use of mechanical and chemical thromboprophylaxis in this large multicenter study. ALIF surgery is thought to carry a higher risk of thromboembolic complications compared with posterior lumbar surgery due to the handling and
Table 2. Indication for procedure.

\begin{tabular}{lcc}
\hline Indication & Number of Patients & Percentage \\
\hline Neuroforaminal stenosis & 59 & 29.5 \\
Spondylolisthesis & 47 & 23.5 \\
Revision discectomy & 45 & 22.5 \\
Degenerative disc disease & 35 & 17.5 \\
Revision for posterior nonunion & 14 & 7 \\
$\quad$ Total & 200 & \\
\hline
\end{tabular}

retraction of the major vessels adjacent to the surgical field during the procedure. ${ }^{10,12-17}$

Unfortunately, the majority of current published literature regarding ALIF surgery does not detail the precise thromboprophylactic regime used. The minority that do detail their regime tend to use some form of mechanical compression device at the time of surgery and immediately postoperation without any form of chemical prophylaxis. ${ }^{14,15}$

$\mathrm{Brau}^{14}$ reported a retrospective analysis of 686 patients undergoing ALIF surgery with a regime of mechanical compression device prophylaxis alone during surgery. No chemical thromboprophylaxis was used nor was routine postoperative DVT screening part of their protocol. They reported a $1.0 \%$ symptomatic DVT rate, of those 7 patients 5 had a below knee DVT and 2 had an above knee DVT. There were no patients recorded with a symptomatic PE. ${ }^{14}$

In a comparable study, Garg et al. ${ }^{15}$ reported 212 ALIF cases and used a similar protocol of a mechanical compression device from surgery until discharge with no chemical thromboprophylaxis. They reported a $2.4 \%$ rate of symptomatic DVT without routine Doppler screening. ${ }^{15}$

There is very limited literature available on centers that use chemical thromboprophylaxis or a combination of mechanical and chemical prophylaxis in ALIF surgery. Mehren et al. ${ }^{18}$ report a retrospective German case series of 120 patients who underwent ALIF surgery. All patients had a postoperative regime of LMWH until fully mobile; no details are provided regarding the use of mechanical thromboprophylaxis, and patients underwent early mobilization from day 1 . With this regime, they reported no instances of significant vessel injury or major bleeding and 1 case of DVT $(0.8 \%){ }^{18}$

One Australian study looking at 188 patients undergoing ALIF or similar total disc replacement lumbar surgery reports using continuous pulse oximeter monitoring on the great toes, if there was any significant reduction in the reading after vessel 
retractor placement, then a single shot of unfractionated heparin was administered intraoperatively to achieve anticoagulation for 30 minutes. In their study of 188 patients, they used heparin in $38.3 \%$ of patients and had 1 patient with a DVT $(0.5 \%) .{ }^{19}$ Overall, they found a small statistically significant increase in the amount of blood loss with those receiving heparin. Our study did not look at the estimated blood loss for each patient; however, increased blood loss does not seem to be a major issue with this regime. There were no episodes of major vessel injury or bleeding, and cell salvage was not required in any of the patients who were included in this study; however, it is important to consider that preoperative anticoagulation could result in difficulty in controlling bleeding, which could occur with the rare complication of major vessel injury. This is a potentially devastating complication, but in the hands of an experienced spine and/or vascular surgeon, the risk should be mitigated through meticulous surgical technique and soft tissue handling. This study has shown a low incidence of vascular injury in the hands of a surgeon performing anterior lumbar spinal surgery regularly $(0 \%)$.

No studies were available looking at aspirin as chemical prophylaxis in ALIF or comparable lumbar disc replacement surgery. However, there is a precedent for aspirin use in some centers for posterior lumbar surgery, as well as other orthopedic surgery. ${ }^{20-22}$

Another major factor in the development of VTE in ALIF surgery is thought to be the method of and duration of vessel retraction during the procedure. Some authors have criticized the use of Steinman pin or fixed abdominal retractor retraction of the vessels due to the perceived increased risk of arterial occlusion, vascular wall injury, and VTE. ${ }^{9,23,24}$ It is the standard practice of the senior author to use Steinman pin retraction of the vessels, and in this study of 200 patients we did not identify any instances of these complications.

The design of this study did not involve routine ultrasound scanning of all patients postoperatively, so it is not possible to comment on the rate of asymptomatic DVT in this patient group. However, the significance of clinically undetectable DVTs remains controversial. The study also did not seek to examine the patients' risk factors for VTE at baseline nor did it look at previous anticoagulation therapy for other medical conditions. The major limitation of this study is the sample size since VTE is a relatively rare complication following ALIF surgery. ${ }^{10,12-16}$

There are a number of facets to the regimen described and understandably there may be concerns regarding potential cost implications. It is only possible to discuss the cost based on a UK healthcare system in the National Health Service. The total cost for the duration of treatment is £146.36, but this does not include the cost of TEDS or pneumatic compression cuffs used. There is significant morbidity associated with VTE along with the potential of an increased/additional length of stay. The exact cost of this is difficult to determine but in terms of increased/added bed stay alone this is around $£ 400$ per day. This does not take into account the cost of ongoing treatment for VTE such as treatment with Factor Xa inhibitors, which costs a minimum of $£ 180$ for a 3 -month course of treatment.

Overall there is a paucity of good evidence regarding the risks and benefits of mechanical and chemical prophylaxis in ALIF surgery. We believe this is the first study to propose a specific regime for the perioperative mechanical and chemical prevention of VTE in patients undergoing anterior lumbar surgery with robust patient numbers and documented efficacy of the regime. It is also in line with the National Institute for Health and Care Excellence guidelines for VTE thromboprophylaxis in spinal surgery and the British Orthopaedic Association and British Association of Spinal Surgeons guidelines for anterior lumbar spine surgery. 4,8

\section{CONCLUSION}

The proposed VTE prophylactic regime is safe and efficacious and may decrease the incidence of symptomatic VTE in patients undergoing an ALIF procedure. Clearly, routine postoperative vein imaging would be needed to confirm the complete absence of VTE using this technique; however, we have demonstrated a reduction in the symptomatic VTE rate following ALIF surgery as compared with the general reported rates in the ALIF literature. In addition to the VTE prophylaxis, the authors believe that that careful patient selection, early mobilization, and minimizing the time the abdominal vessels are under tension are all key factors in reducing the incidence of VTE; although these were not specifically investigated in this study. Furthermore, we believe that despite the use of chemical 
thromboprophylaxis, there is no evidence of bleeding complications as a result of using this regime.

\section{REFERENCES}

1. Fawi HMT, Saba K, Cunningham A, et al. Venous thromboembolism in adult elective spinal surgery. Bone Joint $J$. 2017;99-B(9):1204-1209.

2. Rokito SE, Schwartz MC, Neuwirth MG. Deep vein thrombosis after major reconstructive spinal surgery. Spine. 1996;21(7):853-859.

3. West JL III, Anderson LD. Incidence of deep vein thrombosis in major adult spinal surgery. Spine. 1992;17:254257.

4. National Institute for Health and Care Excellence (NICE). Venous thromboembolism in over 16s: reducing the risk of hospital-acquired deep vein thrombosis or pulmonary embolism. March 2018. www.nice.org.uk/guidance/ng89. Accessed November 4, 2018.

5. Bono CM, Watters WC III, Heggeness MH, et al. An evidence-based clinical guideline for the use of antithrombotic therapies in spine surgery. Spine J. 2009;9(12):1046-1051.

6. North American Spine Society, Committee NE-BGD. North American Spine Society evidence-based clinical guidelines for multidisciplinary spine care: antithrombotic therapies in spine surgery. Published 2009. Accessed August 18, 2018. ht tps://www.spine.org/Portals/0/Documents/ ResearchClinicalCare/Guidelines/AntithromboticTherapies. pdf?ver=2015-06-05-125448-477

7. Brambilla S, Ruosi C, La Maida GA, Caserta S. Prevention of venous thromboembolism in spinal surgery. Eur Spine J. 2004;13(1):1-8.

8. British Orthopaedic Association. BOA VTE living document: specialist society contributions. http://www.boa.ac. uk/wp-content/uploads/2014/01/VTE-Specialist-societies.pdf. Published February 2014. Accessed November 4, 2018.

9. Watkins R. Anterior lumbar interbody fusion surgical complications. Clin Orthop Relat Res. 1992:47-53.

10. Sasso RC, Best NM, Mummaneni PV, Reilly TM, Hussain SM. Analysis of operative complications in a series of 471 anterior lumbar interbody fusion procedures. Spine. 2005;30(6):670-674.

11. Kim JS, Choi KC, Jung B, Lee SH. Thrombosis of left common iliac artery following anterior lumbar interbody fusion: case report and review of literatures. $J$ Korean Neurosurg Soc. 2009;45(4):249-252.

12. Mayer HM. The ALIF concept. Eur Spine $J$. 2000;9(S1):S035-S043.

13. Bateman DK, Millhouse PW, Shahi N, et al. Anterior lumbar spine surgery: a systematic review and meta-analysis of associated complications. Spine J. 2015;15(5):1118-1132.

14. Brau SA. Mini-open approach to the spine for anterior lumbar interbody fusion: description of the procedure, results and complications. Spine J. 2002;2(3):216-223.

15. Garg J, Woo K, Hirsch J, Bruffey JD, Dilley RB.
Vascular complications of exposure for anterior lumbar interbody fusion. J Vasc Surg. 2010;51(4):946-950.

16. Choy W, Barrington N, Garcia RM, et al. Risk factors for medical and surgical complications following single-level ALIF. Global Spine J. 2017;7(2):141-147.

17. Nicol M, Sun Y, Craig N, Wardlaw D. Incidence of thromboembolic complications in lumbar spinal surgery in 1,111 patients. Eur Spine J. 2009;18(10):1548-1552.

18. Mehren C, Mayer HM, Siepe C, Grochulla F, Korge A. The minimally invasive anterolateral approach to L2-L5. Oper Orthop Traumatol. 2010;22(2):221-228.

19. Sim EM, Claydon MH, Parker RM, Malham GM. Brief intraoperative heparinization and blood loss in anterior lumbar spine surgery. J Neurosurgery Spine. 2015;23(3):309-313.

20. Jameson SS, Baker PN, Deehan DJ, Port A, Reed MR. Evidence-base for aspirin as venous thromboembolic prophylaxis following joint replacement. Bone Joint Res. 2014;3(5):146-149.

21. Parvezi J, Ceylan HH, Kicukdurmaz F, Merli G, Tuncay I, Beverland D. Venous thromboembolism following hip and knee arthroplasty, the role of aspirin. J Bone Joint Surg. 2017;99(11):961-972.

22. Ogonda L, Hill J, Doran E, Dennison J, Stevenson M, Beverland D. Aspirin for thromboprophylaxis after primary lower limb arthroplasty: early thromboembolic events and 90 day mortality in 11,459 patients. Bone Joint J. 2016;98$\mathrm{B}(3): 341-348$.

23. Baker JK, Reardon PR, Reardon MJ, Heggeness MH. Vascular injury in anterior lumbar surgery. Spine. 1993;18(15):2227-2230.

24. Ikard RW. Methods and complications of anterior exposure of the thoracic and lumbar spine. Arch Surg. 2006;141(10):1025-1034.

Disclosures and COI: The authors received no funding for this study and report no conflicts of interest.

Corresponding Author: Ata G. Kasis, MD, FRCS(Tr\&Orth), Consultant Trauma and Orthopaedic Spinal Surgeon, Northumbria Healthcare NHS Foundation Trust, Woodhorn Lane, Ashington, NE63 9JJ, United Kingdom. Phone: 01670 529708; Email: ata.Kasis@northumbria-healthcare. nhs.uk.

Published 16 April 2021

This manuscript is generously published free of charge by ISASS, the International Society for the Advancement of Spine Surgery. Copyright (C) 2021 ISASS. To see more or order reprints or permissions, see http://ijssurgery.com. 